Fournal of Hospital Infection (1995) 31, 13-24

REVIEW

\title{
The significance of nasal carriage of Staphylococcus aureus and the incidence of postoperative wound infection
}

\author{
R. P. Wenzel and T. M. Perl \\ Department of Internal Medicine, College of Medicine, University of Iowa, \\ Iowa City, Iowa, USA \\ Accepted for publication 27 April 1995
}

\begin{abstract}
Summary: Staphylococcus aureus infections are associated with considerable morbidity and, in certain situations, mortality. The association between the nasal carriage of $S$. aureus and subsequent infection has been comprehensively established in a variety of clinical settings, in particular, patients undergoing haemodialysis and continuous ambulatory peritoneal dialysis (CAPD), and in patients undergoing surgery. Postoperative wound infections are associated with a high degree of morbidity and represent an important medical issue. Until recently, eradication of $S$. aureus nasal carriage by various topical and systemic agents had proved unsuccessful. Mupirocin is a novel topical antibiotic with excellent antibacterial activity against staphylococci. Recent studies have demonstrated that intranasal administration of mupirocin is effective in eradicating the nasal carriage of $S$. aureus and in reducing the incidence of $S$. aureus infections in haemodialysis and CAPD patients. It has been suggested that sufficient evidence now exists to test the hypothesis that eradication of the carrier state in surgical patients preoperatively may reduce the incidence of $S$. aureus postoperative wound infections.
\end{abstract}

Keyzoords: Staphylococcus aureus: surgery; wound infection; mupirocin.

\section{Introduction}

Staphylococcus aureus is one of the most important causes of nosocomialand community-acquired infections. Although staphylococci are common inhabitants of the skin and the mucous membranes, the anterior nares provide the principal reservoir for these organisms. The general population, health care workers and patients can become intermittent or persistent nasal carriers of $S$. aureus, although the prevalence of nasal carriage varies widely depending upon the population. ${ }^{1}$ Studies have shown that $10-15 \%$ of

Correspondence to: Professor R. P. Wenzel, Division of General Medicine, Clinical Epidemiology and Health Services Research, Department of Internal Medicine, College of Medicine, University of Iowa, Iowa City, Iowa 52242, USA. 
healthy adults carry $S$. aureus in their nares; this figure rises to approximately 20-35\% in hospital personnel. ${ }^{2}$ Many patient groups have higher rates of $S$. aureus nasal carriage in comparison to the general population and health care workers, such as patients infected with HIV and those treated by haemodialysis and continuous ambulatory peritoneal dialysis (CAPD)..$^{3-6}$

The postulated sequence of events which leads to infection is initiated with $S$. aureus nasal carriage. The organisms are then disseminated via hand carriage to other body sites where infection can occur when breaks in the dermal surfaces, by vascular catheterization or surgical incisions, have occurred. S. aureus is the most common organism responsible for postoperative wound infections and a leading cause of septicaemia, intravenous catheter-related infections and skin and soft-tissue infections.

Auto-infection of surgical wounds by $S$. aureus is common, and is associated with considerable morbidity and represents an important medical and economic problem. To date, no comprehensive studies have been undertaken to demonstrate whether the eradication of the carrier state would reduce the rate of wound infections by $S$. aureus. However, recent studies in patients undergoing haemodialysis ${ }^{6}$ and $\mathrm{CAPD}^{7}$ have shown that eradication of nasal carriage of $S$. aureus significantly reduces the incidence of infection in these patients. The evidence, therefore, suggests that eradication of the carrier state in patients preoperatively may reduce the rate of postoperative wound infections with $S$. aureus. ${ }^{8}$ Indeed, this idea has been supported by a study, with historical controls, recently carried out by Kluytmans et al. ${ }^{9}$

\section{Staphylococcus aureus nasal carriage and infection}

The significance of nasal carriage of staphylococci in the epidemiology of staphyloccocal infection has been recognized for 40 years. ${ }^{1}$ Tulloch ${ }^{10}$ reportcd that staphylococci isolated from skin lesions in patients with chronic staphylococcal skin infection were of the same phage-type as staphylococci isolated from the anterior nares of patients in $88 \%$ of instances in which staphylococci were typable.

\section{Infection in patients undergoing haemodialysis}

Infection in haemodialysis patients is associated with considerable morbidity and is the second leading cause of mortality in this group of patients. The most common site of infection is that related to the vascular access site, and studies have shown that $S$. aureus accounts for $70-92 \%$ of these infections. ${ }^{11} S$. aureus nasal carriage in haemodialysis patients is relatively high, with up to $70 \%$ of patients carrying $S$. aureus in their anterior nares. ${ }^{6}$ A direct link between nasal carriage of $S$. aureus and subsequent infections by the same organism was demonstrated in a five year prospective study of haemodialysis patients. ${ }^{6}$ Yu et al. ${ }^{6}$ found that of 16 dialysis patients with S. aureus infections, 14 were nasal carriers of staphylococci. Moreover, 93\% 
$(13 / 14)$ of $S$. aureus infected carriers were found to be infected with the same phage-type as that carried in their nares. Importantly, patients who were nasal carriers of $S$. aureus had a significantly higher incidence of staphylococci infections than non-carriers. Similarly, in a study carried out by Ena et al..$^{12}$ patients who developed staphylococcal infections while undergoing haemodialysis were found to be infected by strains persistently carried in their nares.

\section{Infection in patients undergoing $C A P D$}

Studies evaluating patients on long-term CAPD have demonstrated the association between nasal carriage of $S$. aureus and subsequent infection in CAPD patients. ${ }^{13}$ Peritonitis and exit-site infections are major complications of CAPD and are a leading cause of mortality in this group of patients. Sewell et al..$^{13}$ studied 30 patients, of which four were chronic $S$. aureus carriers, six intermittent carriers and 20 non-carriers. Of the chronic carriers, three patients developed five episodes of exit-site infections and six episodes of peritonitis, whereas three of the intermittent carriers developed three episodes of exit-site infections and one episode of peritonitis. In contrast, only four of the 20 non-carriers developed four episodes of exit-site infections and one of peritonitis. Chronic carriers, therefore, appear to be at a higher risk of developing $S$. aureus-related infections. In a subsequent study by Kim et al. ${ }^{14} 10$ of 12 CAPD patients were found to have isolates of the same phage-type in cultures of the exit-site and peritoneal fluid. Furthermore, of the 12 patients, seven had isolates with the same phage-type in cultures from the nares, hands and peritoneal fluid.

In a study in which the number of exit-site infections in 87 CAPD patients was recorded over eight months, patients who were nasal carriers were found to have a 6.7 -fold increase in the incidence of exit-site infections compared with patients with negative cultures of the nares. ${ }^{5}$ In a similar study in which 43 patients, 16 chronic carriers and 12 intermittent carriers, underwent CAPD prospectively for 15 months, 16 episodes of peritonitis occurred in the carrier group compared with none in the non-car rier gruup. Moreover, of those patients evaluated, $100 \%$ of strains isolated from the nares and the peritoneum were found to be of identical plasmid pattern. ${ }^{15}$

In a study carried out by Luzar et al. ${ }^{4}$ the relationship between the nasal carriage of $S$. aureus and subsequent catheter exit-site infection or peritonitis was assessed in 140 consecutive patients who were new to CAPD. Of these patients 63 were nasal carriers at the initiation of treatment. Luzar et al. ${ }^{4}$ found a fourfold higher incidence of exit-site infections in nasal carriers in comparison with non-carriers. The probability of remaining free of an exitsite infection after 18 months on CAPD was $92 \%$ among non-carriers and $54 \%$ in carriers $(P=0.012)$. Furthermore, among nasal carriers, 11 of 31 episodes of peritonitis were caused by $S$. aureus, compared with none of 36 episodes in the non-carriers. 
Methicillin-resistant $S$. aureus (MRSA) nasal carriage in patients undergoing CAPD is associated with an increased risk of CAPD-related infections in comparison with methicillin-susceptible $S$. aureus (MSSA) nasal carriers and non-carriers. ${ }^{16}$ In a recent study, patients who were MRSA carriers were found to have an increased rate of exit-site infections and peritonitis. The risk of being free from MRSA peritonitis after one year on CAPD in the carrier group was 65 vs. $96 \%$ in the non-carrier group $(P<0 \cdot 01)$. Similarly, the risk of being free from an exit-site infection after one year on CAPD was $67 \%$ in the carrier group in comparison with $96 \%$ in the non-carrier group $(P<0 \cdot 01){ }^{16}$ The number of patients who had a combination of CAPD-related infections or multiple episodes of peritonitis was also significantly higher in the carrier group than in the non-carrier group $(P<0.001)$. Moreover, MRSA nasal carriers suffered an increased number of catheter losses and CAPD drop-outs in comparison to MSSA carriers and non-carriers. ${ }^{16}$

\section{Infection in $H I V$-infected and AIDS patients}

Recent studies have demonstrated an increased prevalence of $S$. aureus carriage among $\mathrm{HIV}$-infected patients ${ }^{3,17}$ and patients with AIDS. ${ }^{18}$ In a study by Weinke $e t a l,{ }^{3}$ the frequency of nasopharyngeal $S$. aureus carriage in HIV patients and its relationship to the incidence of $S$. aureus septicaemia was examined. The study, conducted over eight months, found that $44 \%$ $(60 / 136)$ of HIV-infected patients were nasal carriers of $S$. aureus. In comparison only $31 \%(12 / 39)$ patients with chronic disease and $23 \%(11 /$ 47 ) healthy hospital staff demonstrated $S$. aureus nasal carriage. Of eight HIV-infected patients with $S$. aureus septicaemia all were found to be nasal carriers. In contrast, no episodes of septicaemia occurred in non-colonized patients $(P<0 \cdot 01)$.

\section{Postoperative zound infections}

Postoperative wound infections affect at least 920000 of the 23 million patients who undergo surgery each year in the USA. ${ }^{19}$ Surgical site infections are associated with high degree of morbidity and have important medical consequences in terms of prevention and treatment. S. aureus can account for up to $19 \%$ of postoperative wound infections in patients who are nasal carriers of $S$. aureus ${ }^{20}$ (Table I). For several decades, it has been known that $S$.aureus nasal carriage by patients predisposes to postoperative wound auto-infection. Auto-infection of surgical wounds by $S$. aureus was first documented in $1959^{21,22}$ and the early $1960 \mathrm{~s} .^{20,23-27}$ In a study of 125 patients undergoing major surgery, patients with positive preoperative $S$. aureus nasal cultures demonstrated a postoperative wound infection rate of $37 \%$. In comparison, those patients with negative preoperative nasal cultures demonstrated an $11 \%$ infection rate. ${ }^{21}$ Moreover, of those patients who were staphylococcal nasal carriers, S. aureus was the organism isolated from $94 \%$ of postoperative wound cultures. In contrast, a variety of organisms were 
Table I. Incidence of postoperative wound infection in nasal carriers and non-carriers of Staphylococcus aurcus

\begin{tabular}{lcccc}
\hline & & \multicolumn{2}{c}{ Incidence of wound infection } & \\
\cline { 3 - 4 } First author & $\begin{array}{c}\text { Year of } \\
\text { report }\end{array}$ & $\begin{array}{c}\text { No. infected/ } \\
\text { no. colonized }\end{array}$ & $\begin{array}{c}\text { No. infected/ } \\
\text { no. not colonized }\end{array}$ & $\begin{array}{c}\text { \% } \\
\text { Endogenous* }\end{array}$ \\
\hline White $^{20}$ & 1964 & $20 / 106(19 \%)$ & $28 / 345(8 \%)$ & 66 \\
Williams $^{22}$ & 1959 & $20 / 276(7 \%)$ & $7 / 342(2 \%)$ & 55 \\
Public Health $_{\text {Laboratory }}^{23}$ & 1960 & $73 / 821(9 \%)$ & $158 / 2235(7 \%)$ & 33 \\
McNeill $^{24}$ & 1961 & $12 / 74(16 \%)$ & $11 / 113(10 \%)$ & 42 \\
Henderson $^{25}$ & 1961 & $22 / 264(8 \%)$ & $18 / 569(3 \%)$ & 30 \\
Bassett $^{26}$ & 1963 & $24 / 442(5 \%)$ & $6 / 78(8 \%)$ & 58 \\
Calia $^{27}$ & 1969 & $16 / 96(17 \%)$ & $16 / 173(9 \%)$ & 100 \\
\hline
\end{tabular}

* By phage-typing-showing same strains in preoperative nasal culture as identified in postoperative wound infection.

isolated from postoperative wound cultures in non-carriers. Furthermore, $92 \%$ of nasal carriers with postoperative staphylococcal wound infections demonstrated identical $S$. aureus phage-types from both nose and wound cultures.

Subsequent studies have shown the incidence of surgical wound sepsis in nasal carriers of $S$. aureus ranges from $5-19 \%$ (Table I). Moreover, $30-100 \%$ of wounds were infected with phage-types that were indistinguishable from those in the patient's nose. In comparison, the postoperative infection rate in non-carriers was between $2-10 \%$ (Table I).

\section{Eradication of Staphylococcus aureus nasal carriage and the reduction in the incidence of infections}

The link between nasal carriage of $S$. aureus and the aetiology of subsequent infections caused by this organism is well established in both haemodialysis/ CAPD patients and surgical patients. However, eradication of the carrier state in patients prior to surgery, and the concomitant reduction in postoperative wound infections with $S$. aureus, have yet to be fully evaluated. ${ }^{8}$ Mupirocin (pseudomonic acid) has been shown to have good in-vitro activity against clinical isolates of $S$. aureus, ${ }^{28}$ and the efficacy of mupirocin in eradicating nasal $S$. aureus carriage in haemodialysis patients and healthy control volunteers has been comprehensively evaluated in several studies. ${ }^{29-33}$ The use of mupirocin has also been advocated to eradicate MRSA nasal colonization in hospital staff and patients in the control of epidemic MRSA. ${ }^{34,35}$ Moreover, recent studies have demonstrated the efficacy of mupirocin, in reducing both the carriage and the incidence of $S$. aureus infection among patients undergoing haemodialysis and CAPD. ${ }^{7,36}$ 


\section{Haemodialysis and CAPD patients}

Several studies carried out by Boelaert et al. ${ }^{36,37}$ and Holton et al..$^{38}$ have evaluated the efficacy of mupirocin in eradicating $S$. aureus nasal carriage and reducing the incidence of $S$. aureus infections in haemodialysis patients. In a prospective, double-blind, placebo-controlled trial of 34 nasal carriers, intranasal application of $2 \%$ mupirocin ointment three times daily for two weeks followed by three times weekly applications for nine months, significantly reduced the incidence of $S$. aureus nasal carriage. Indeed, only $6 \%$ of nasal cultures in the mupirocin arm of the study were positive for $S$. aureus in comparison with $58 \%$ in the placebo arm of the study. There was also a significant reduction in the incidence of $S$. aureus infections, with one episode in the mupirocin group in comparison with six in the placebo group. ${ }^{36} \mathrm{~A}$ subsequent study by Boelaert et $a l .{ }^{39}$ demonstrated that continuous therapy with mupirocin was superior to intermittent therapy.

The efficacy of mupirocin in reducing the incidence of $S$. aureusassociated bacteraemias was also evaluated by Boelaert et al.$^{37}$ in patients undergoing haemodialysis. In a prospective study, Boelaert et al.$^{37}$ applied $2 \%$ calcium mupirocin to stable $S$. aureus nasal carriers in the haemodialysis unit, three times daily for five days then three times weekly for six months and thereafter once weekly for the subsequent 18 months of the study. This regimen eradicated nasal $S$. aureus carriage in $96.3 \%$ of surveillance cultures and reduced fourfold the incidence of $S$. aureus bacteraemia per patient year among all dialysis patients. $S$. aureus was responsible for four out of 23 episodes of bacteraemia (17.4\%) following mupirocin treatment. In comparison, the incidence of $S$. aureus bacteraemia assessed from historical controls was reported to be 18 out of 33 bacteraemia episodes $(54.5 \%)$. Eradication of $S$. aureus from the nares did not lead to overgrowth by other organisms and the authors concluded that nasal mupirocin was effective in eradicating nasal carriage of $S$. aureus and decreasing the incidence of bacteraemias caused by this pathogen.

The findings of Boelaert et al.$^{36,37}$ were supported in a study carried out by Holton et al. ${ }^{38}$ in which nasal mupirocin was applied to the anterior nares of patients undergoing haemodialysis. Holton et al. ${ }^{38}$ treated 22 haemodialysis patients who were identified as $S$. aureus nasal carriers. The eradication rate immediately following the completion of therapy was $77 \%$ $(17 / 22)$. Of the 17 patients who were culture-negative at completion of therapy, all remained free from $S$. aureus infections at three months followup. In contrast, two out of five patients $(40 \%)$ who remained nasal culturepositive following mupirocin treatment suffered a $S$. aureus infection. Ten out of 46 patients $(22 \%)$ not enrolled in the study but followed concurrently had a significantly higher incidence of $S$. aureus infection $(P=0.03)$.

In a recent study, the efficacy of mupirocin in the eradication of nasal $S$. aureus in CAPD patients and the reduction in the incidence of CAPDrelated infections was evaluated. ${ }^{7}$ Ninety-four CAPD patients were enrolled in the study and of these $47 \cdot 5 \%$ were $S$. aureus nasal carriers. A retrospective 
assessment of 74 CAPD patients served as historical controls. Two percent mupirocin ointment, applied three times a day for seven days to the patients' nares, eradicated nasal $S$. aureus carriage in $100 \%$ of patients. The incidence of $S$. aureus-related peritonitis and exit-site infections was also significantly reduced following mupirocin treatment. Only two episodes of peritonitis occurred in CAPD patients, in contrast to 18 episodes in historical controls. Fxit-site infections accounted for eight and 19 episodes in CAPD patients and historical controls, respectively.

\section{Surgical patients}

The efficacy of mupirocin in the reduction of both $S$. aureus nasal carriage and subsequent infection in patients undergoing haemodialysis and CAPD has prompted Wenzel ${ }^{8}$ to suggest that sufficient evidence now exists lo test the hypothesis that eradication of the carrier state would reduce the incidence of postoperative wound infections with $S$. aureus. Indeed, this has been reported recently in a historically controlled study conducted by Kluytmans et $a l .{ }^{9}$ In this study, mupirocin nasal ointment was applied to the nose twice daily for five days, commencing one day prior to the operation, in 629 patients undergoing cardiothoracic surgery during a 12 month period. The overall postoperative rate of infection assessed from 983 historical controls was $8.9 \%$, and of these $3.5 \%$ were caused by $S$. aureus. In comparison, the incidence of all postoperative wound infections, and in particular those caused by $S$. aureus were significantly reduced during the period of mupirocin treatment, with figures of $1.8 \%(P<0 \cdot 0001)$ and $0.6 \%$ $(P<0 \cdot 0005)$, respectively. $S$. aureus was isolated from four patients with postoperative wound infections following mupirocin treatment. However, the preoperative nasal cultures from these four patients did not grow $S$. aureus. Phage-typing showed that two of the isolates were identical to the type isolated previously from the nose of one of the nurses on the ward. Possibly, the infection was acquired postoperatively in the ward. Results, therefore, suggest that preoperative application of mupirocin reduces nasal carriage and reduces the incidence of postoperative wound infections by $S$. aureus. However, it must be remembered that this conclusion is based on only one historically controlled study. Large randomized, placebocontrolled clinical trials are required to assess more fully the efficacy of applying mupirocin preoperatively to reduce the incidence of postoperative wound infections. One such clinical trial is underway at the College of Medicine, University of Iowa, USA.

\section{Resistance}

The topical use of any antibiotic is invariably associated with concerns about the emergence of resistance. ${ }^{39}$ Mupirocin-resistant $S$. aureus have rarely, however, been encountered in patients treated with mupirocin to prevent haemodialysis or peritoneal dialysis-associated infections. In a nine 
month study by Boelaert et al. ${ }^{36}$ mupirocin was applied to the anterior nares of stable $S$. aureus nasal carriers undergoing haemodialysis; three times a day for two weeks followed by three times a week for the remaining nine months. Throughout the study all $S$. aureus isolates remained mupirocin sensitive [minimum inhibitory concentration (MIC) $\leq 1 \mathrm{mg} / \mathrm{L}$ ]. In a subsequent study of haemodialysis patients with stable $S$. aureus nasal carriage, mupirocin was applied to the anterior nares three times a day for five days, then three times weekly for six months and thereafter once weekly for the subsequent 18 months of the study. Among the 29 S. aureus strains recovered from subsequent surveillance cultures of the nares, only one mupirocin-resistant strain was found (MIC $>512 \mu \mathrm{g} / \mathrm{mL}$ ), ${ }^{37}$ i.e. one resistant isolate over 108 patient years. Further studies by Boelaert et $a l^{40,41}$ on the application of mupirocin to haemodialysis paticnts who were $S$. aureus nasal carriers have not demonstrated the development of mupirocin resistance.

Low- and high-level resistance by $S$. aureus to mupirocin has, however, been reported in the UK and is well documented in several letters to journals ${ }^{42-45}$ and in the general literature. ${ }^{46,47}$ Despite this, resistance among $S$. aureus to mupirocin remains uncommon. A multi-centre UK survey found only $0.3 \%$ of $7137 \mathrm{~S}$. aureus isolates to be resistant to mupirocin $(\mathrm{MIC}>4 \mathrm{mg} / \mathrm{L})$ and only four of these 23 isolates $(17 \%)$ were highly resistant (MIC $>512 \mathrm{mg} / \mathrm{L}) .^{44}$ In a separate study, during a MRSA outbreak in a Spanish hospital, an extensive screening programme combined with the use of intranasal mupirocin brought a two-year MRSA outbreak under control, 53 of 530 patients $(10 \%)$ carried MRSA with low-level resistance to mupirocin (MICs $8-32 \mathrm{mg} / \mathrm{L}$ ), of which $38(72 \%)$ had previously had mupirocin-sensitive strains. ${ }^{48}$

The overall incidence of $S$. aureus strains showing high-level resistance to mupirocin (MICs $>512 \mathrm{mg} / \mathrm{L}$ ) remains extremely low, despite increasing worldwide usage of mupirocin. Where high level resistance to mupirocin has been encountered, such strains have generally been associated with patients on dermatology wards $\mathrm{s}^{42,43,45}$ or others receiving long-term mupirocin thcrapy of scveral wecks in duration. ${ }^{44}$ In this setting, it has been suggested that there may be an environmental reservoir contributing to the spread of mupirocin-resistant isolates. ${ }^{49}$

Since the development of resistance to mupirocin is a cause for concern and the use of mupirocin preoperatively may encourage the emergence of mupirocin-resistant MRSA, alternative topical and systemic agents are available which can be used to eradicate the nasal carriage of $S$. aureus with varying degrees of success. Since it is not the intention of this paper to review alternative agents, readers are referred to a review of the subject by Hudson. ${ }^{50}$

\section{Dosing regimen for intranasal mupirocin}

The duration and frequency of mupirocin dosing have varied between studies. Overall the clinical data support twice daily use of intranasal 
mupirocin for five days. Minimal dose requirements, to reduce the emergence of mupirocin resistance, have been investigated by Casewell and $\mathrm{Hill}^{51}$ in 44 stable nasal carriers of MSSA. A single dose of mupirocin, or a regimen of four times daily for two days, eliminated nasal carriage of $S$. aureus within $24 \mathrm{~h}$. Seven days after the single dose and the two day course, 92 and $96 \%$ of subjects, respectively, remained free of nasal $S$. aureus carriage. Recently, data from a one year prospective cohort study of $S$. aureus nasal carriers given treatment with mupirocin or placebo were reported. ${ }^{52}$ All subjects received only five days of active drug or placebo. At six months, nasal carriage for $S$. aureus was $48 \%$ in the treatment group vs. $72 \%$ in controls $(P=0.054)$. At one year, nasal carriage was 53 vs. $76 \%$, respectively $(P=0 \cdot 056)$. 'Thus, a single brief treatment course of topical nasal mupirocin reduced nasal carriage for up to one year. Therefore, it would seem appropriate to recommend the use of mupirocin for five days, particularly in cases of MRSA.

\section{Conclusion}

Staphylococcus aureus remains a leading cause of infection in several clinical areas. In particular, $S$. aureus is responsible for a large proportion of postoperative wound infections and infections in patients undergoing haemodialysis or peritoneal dialysis. The significance of nasal carriage of $S$. aureus in the epidemiology of infection has been comprehensively established. Indeed, nasal carriage of $S$. aureus predisposes patients to autoinfection. Postoperative wound infections are associated with considerable morbidity and place a substantial medical and economic burden on both the patient and hospital. Between 30 and $100 \%$ of postoperative wound sepsis can be the result of endogenous strains of $S$. aureus. The reduction or prevention of postoperative wound infection by $S$. aureus is, therefore, an important medical goal. Elimination of nasal carriage of $S$, aureus can be achicved by various systemic and topical antibodics: howcrer, topical mupirocin has been shown to be particularly effective. The eradication of the nasal carriage of $S$. aureus in haemodialysis and CAPD patients is associated with a significant reduction in the incidence of infections. Extrapolation from these results would suggest that elimination of nasal carriage of $S$. aureus in patients undergoing surgical procedures might reduce the incidence of postoperative wound infections due to this organism. This hypothesis has been examined in a historically controlled clinical trial carried out by Kluytmans et al. ${ }^{9}$ in the Netherlands, and the results suggested a reduction in postoperative wound infections following eradication of $S$. aureus nasal carriage preoperatively. To corroborate these results, a large, randomized, placebo-controlled clinical trial is underway at the College of Medicine, University of Iowa, USA. 


\section{References}

1. Casewell MW, Hill RLR. The carrier state: methicillin-resistant Staphylococcus aureus. F Antimicrob Chemother 1986; 18: 1-12.

2. Willems FThC. Epidemiology of nasal carriage of Staphylococcus aureus. In: van der Meer JWM, Ed. Nasal Carriage of Staphylococcus aureus: A Round Table Discussion. Excerpta Medica 1990; 3-6.

3. Weinke T, Schiller R, Fehrenbach FJ, Pohle HD. Association between Staphylococcus aureus nasopharyngeal colonisation and septicaemia in patients infected with the human immunodeficiency virus. Eur $\mathcal{F}$ Clin Microbiol Infect Dis 1992; 11: 985-989.

4. Luzar MA, Coles GA, Faller B et al. Staphylococcus aureus nasal carriage and infection in patients on continuous ambulatory peritoneal dialysis. $N$ Engl $\mathcal{F}$ Med 1990; 322: 505-509.

5. Davies SJ, Ogg CS, Cameron JS, Poston S, Noble WC. Staphylococcus aureus nasal carriage, exit-site infection and catheter loss in patients treated with continuous ambulatory peritoneal dialysis. Peritoneal Dial Int 1989; 9: 61-64.

6. Yu VL, Goetz A, Wagener M et al. Staphylococcus aureus nasal carriage and infection in patients on hemodialysis. N Engl I Med 1986; 315: 91-96.

7. Pérez-Fontán M, García-Falcón T, Rosales M et al. Treatment of Staphylococcus aureus nasal carriers in continuous ambulatory peritoneal dialysis with mupirocin: long-term results. Am F Kidney Dis 1993; 22: 709-712.

8. Wenzel RP. Preoperative antibiotic prophylaxis. $N$ Engl $\mathcal{J}$ Med 1992; 326: 337-339.

9. Kluytmans JAJW, Mouton JW, Ijzerman EPF et al. Nasal carriage of Staphylococcus aureus as a major risk factor for wound infections after cardiac surgery. I Infect Dis 1995; 171: 216-217.

10. Tulloch LG. Nasal carriage in staphylococcal skin infections. BMF 1954; 2: 912-913.

11. Chow JW, Yu VL. Staphylococcus aureus nasal carriage in hemodialysis patients. Arch Intern Med 1989: 149: 1258-1262.

12. Ena J, Boelaert JR, Boyken L, Van Landuit HW, Godard HW, Herwaldt LA. Epidemiology of infections in nasal carriers of Staphylococcus aureus on hemodialysis. In: Proceedings of the 31st Interscience Conference on Antimicrobial Agents and Chemotherapy 1991; Abstract no. 28: 103.

13. Sewell CJ, Clarridge J, Lacke C, Weinman EJ, Young EJ. Staphylococcal carriage and subsequent infection in peritoneal dialysis patients. F Am Med Assoc 1982; 248: 1493-1495.

14. Kim D, Tapson J, Khanna R, Vas SI, Oreopoulos DG. Staphylococcus aureus in patients on continuous ambulatory peritoneal dialysis. Trans Am Soc Artif Intern Organs 1984; 30: 494.

15. Sesso R, Draibe S, Castelo A, Sato I, Leme I, Barbosa D, Ramos O. Staphylococcus aureus skin carriage and development of peritonitis in patients on continuous ambulatory peritoneal dialysis. Clin Nephrol 1989; 31: 264-268.

16. Lye WC, Leong SO, Lee EJC. Methicillin-resistant Staphylococcus aureus nasal carriage and infection in CAPD. Kidney Int 1993; 43: 1357-1362.

17. Ganesh R, Castle D, McGibbon D, Phillips I, Bradbeer C. Staphylococcal carriage and HIV infection. Lancet 1989; ii: 558.

18. Jacobson MA, Gellermann H, Chambers H. Staphylococcus aureus bacteremia and recurrent staphylococcal infection in patients with acquired immunodeficiency syndrome and AIDS-related complex. Am $\mathscr{F}$ Med 1988; 85: 172-176.

19. Haley RW, Culver DH, Morgan WM, White JW, Emori TG, Hooton TM. Identifying patients at high risk of surgical wound infection: a simple multivariate index of patients susceptibility and wound contamination. Am $\mathscr{F}$ Epidemiol 1985; 121: 206-215.

20. White A. Increased infection rates in heavy nasal carriers of coagulase-positive staphylococci. Antimicrob Agents Chemother 1964; 1963: 667-670.

21. Weinstein HJ. The relationship between the nasal staphylococcal carrier state and the incidence of post-operative complications. $N$ Engl of Med 1959; 260: 1303-1308.

22. Williams REO, Jevons MP, Shooter RA et al. Nasal staphylococci and sepsis in hospital patients $B M \mathcal{F} 1959 ; 2: 658-662$.

23. Incidenec of surgical wound infection in England and Wales. $\Lambda$ report of the Public Health Laboratory Service. Lancet 1960; 2: 659-663. 
24. McNeill IF, Porter IA, Green CA. Staphylococcal infection in a surgical ward. BMF 1961; 2: 798-802.

25. Henderson RJ, Williams REO. Nasal disinfection in prevention of postoperative staphylococcal infection of wounds. BMY 1961;2: 330-333.

26. Bassett HFM, Ferguson WG, Hoffman E, Walton M, Blowers R, Conn CA. Sources of staphylococcal infection in surgical wound sepsis. F Hygiene 1963; 61: 83-94.

27. Calia FM, Wolinsky E, Mortimer EA Jr, Rammelkamp CH Jr. Importance of the carrier state as a source of Staphylococcus aureus in wound sepsis. F Hygiene 1969; 67: $49-57$.

28. Casewell MW, Hill RLR. In vitro activity of mupirocin ('pseudomonic acid') against clinical isolates of Staphylococcus aureus. F Antimicrob Chemother 1985; 15: 523-531.

29. Hingst V, Vergetis W, Bommer J, Borneff M. Prospective randomised placebo-controlled study concerning the elimination of Staphylococcus aureus by means of mupirocin in patients undergoing hemodialysis (poster). International Congress on Management of Infection. Amsterdam. 5-9 April 1992; Abstract p2: 110.

30. Watanakunakorn C, Brandt J, Durkin P, Santore S, Bota B, Stahl CJ. The efficacy of mupirocin ointment and chlorhexidine body scrubs in the eradication of nasal carriage of Staphylococcus aureus among patients undergoing long-term haemodialysis. Am $\mathcal{F}$ Infect Control 1992; 20: 138-141.

31. Muro K, Lim PB. A comparison of mupirocin and rifampicin in short term eradication of Staphylococcus aureus nasal carriage in hemodialysis patients. F Am Soc Nephrol 1991; 2: 340 .

32. Reagan DR, Doebbeling BN, Pfaller MA et al. Elimination of coincident Staphylococcus aureus nasal and hand carriage with intranasal application of mupirocin calcium ointment. Ann Intern Med 1991; 114: 101-106.

33. Casewell MW, Hill RLR. Elimination of nasal carriage of Staphylococcus aureus with mupirocin (psueudomonic acid')—a controlled trial. Fै Antimicrob Chemother 1986; 17: $365-372$.

34. Report of a Combined Working Party of the Hospital Infection Society and British Society for Antimicrobial Chemotherapy. Guidelines for the control of epidemic methicillin-resistant Staphylococcus aureus. F Hosp Infect 1986; 7: 193-201.

35. Hill RLR, Duckworth GJ, Casewell MW. Elimination of nasal carriage of methicillinresistant Staphylococcus aureus with mupirocin during a large hospital outbreak. $\mathcal{F}$ Antimicrob Chemother 1988; 22: 377-384.

36. Boelaert JR, De Smedt RA, Baere YA et al. The influence of calcium mupirocin nasal ointment of the incidence of Staphylococcus aureus infections in haemodialysis patients. Nephrol Dial Transplant 1989; 4: 278-281.

37. Boelaert JR, Van Landuyt HW, Godard CA et al. Nasal mupirocin ointment decreases the incidence of Staphylococcus aureus bacteraemias in haemodialysis patients. Nephrol Dial Transplant 1993; 8: 235-239.

38. Holton DL, Nicolle LE, Diley D, Bernstein K. Efficacy of mupirocin nasal ointment in eradication Staphylococcus aureus nasal carriage in chronic haemodialysis patients. $\mathcal{F}$ Hosp Infect 1991; 17: 133-137.

39. Neu HC. The use of mupirocin in controlling methicillin-resistant Staphylococcus aureus. Infect Control Hosp Epidemiol 1990; 11: 11-12.

40. Boelaert JR, De Baere YA, Godard C, Van Landuyt HW. Eradication of Staphylococcus aureus in dialysis by nasal mupirocin. In: Proceedings of the 29th Interscience Conference on Antimicrobial Agents and Chemotherapy, Houston, 1989; Abstract no. 1262: 315.

41. Boelaert JR, De Baere YA, Geernaert MA, Godard CA, Van Landuyt HW. The use of nasal mupirocin ointment to prevent Staphylococcus aureus bacteraemias in haemodialysis patients: an analysis of cost-effectiveness. F Hosp Infert 1991; 19: 41-46

42. Rahman M, Noble WC, Cookson B. Mupirocin-resistant Staphylococcus aureus. Lancet 1987; 2: 387.

43. Smith GE, Kennedy CTC. Staphylococcus aureus resistant to mupirocin. $\mathscr{F}$ Antimicrob Chemother, 1988; 21: 141-142.

44. Cookson BD, Lacey RW, Noble WC, Reeves DS, Wise R, Redhead RJ. Mupirocinresistant Staphylococcus aureus. Lancet 1990; 335: 1095-1096.

45. Baird D, Coia J. Mupirocin-resistant Staphylococcus aureus. Lancet 1987; 2: 387-388.

46. Cookson BD. Mupirucin resistance in staphylococci. ₹ै Antimicrob Chemother 1990; 25 : 497-503. 
47. Gilbart J, Perry CR, Slocombe B. High level mupirocin resistance in Staphylococcus aureus: evidence for two distinct isoleucyl-tRNA synthetase. Antimicrob Agents Chemother 1993; 37: 32-38.

48. Coell R, Gaspar C, Fernandez C, Arroyo P, Cruzet F. The importance of detecting asymptomatic carriage and the use of nasal mupirocin for the control of methicillinresistant Staphylococcus aureus. In: Proceedings of the 32nd Interscience Conference on Antimicrobial Agents and Chemotherapy, 11-14 October 1992, Anaheim; Abstract no. 366: 172.

49. Layton MC, Perez M, Heald P, Patterson JE. An outbreak of mupirocin-resistant Staphylococcus aureus on a dermatology ward associated with an enivornmental reservoir. Infect Control Hosp Epidemiol 1993; 14: 369-375.

50. Hudson I. The efficacy of intranasal mupirocin in the prevention of staphylococcal infections: a review of recent experience. F Hosp Infect 1994; 27: 81-98.

51. Casewell MW, Hill RLR. Minimal dose requirements for nasal mupirocin and its role in the control of epidemic MRSA. F Hosp Infect 1991; 19 Suppl. B: 35-40.

52. Doebbeling BN, Reagan DR, Pfaller MA, Houston AK, Hollis RJ, Wenzel RP. Long-term efficacy of intranasal mupirocin ointment: a prospective cohort study of Staphylococcus aureus carriage. Arch Intern Med 1994; 154: 1505-1508. 